\title{
ABSTRACTS OF ACC CONFERENCE PROCEEDINGS
}

\section{Poster Presentations}

\section{An anatomical study of arcuate foramina \\ Salman Afsharpour, Kathryn Hoiriis, Bruce Fox, Samuel Demons}

Background: Certain anomalies of the atlas vertebra may have clinical significance; one of these is a posterolateral bony bridge forming an arcuate foramen. A wide range of prevalence and incidence was found in the literature. Objectives: The objective was to study the relationship of the vertebral artery to the atlas in the suboccipital region using cadavers. Methods: Out of 40, 1 male cadaver presented the anomalies of complete bilateral arcuate foramen. Visual observations, measurements, and radiographic examinations were performed. Results: The anomaly/variation was bilateral and almost symmetrical. The vertebral arteries were found passing through a bony tunnel formed by double foramina (lateral and dorsal) on each side. Discussion: This study found a variation of the anomaly in which the vertebral artery was in a protected position. Pressure will not be able to compress the vertebral arteries in this rare type of anomaly. Conclusions: Arcuate foramina have been shown highly variable in shape and size, not only in different people but also in the same individual. The suboccipital region should be evaluated in patients with upper cervical complaints for presence of complete or incomplete arcuate foramen. (This is a conference presentation abstract and not a full work that has been published.)

\section{The prevalence of wrist ganglia in a chiropractic student population}

Caleb Alford, Kevin Bradshaw, Eric Michener, Aaron Perkins, Kayla St. Aubin, Daniel Haun

Objective: Ganglion cysts of the wrists are a common and mostly asymptomatic occurrence, but they can become a symptomatic. Wrist ganglia may be more common in people who perform repetitive manual tasks, such as chiropractic interventions. The objective was to establish the prevalence of wrist ganglia in a population of chiropractic students. Methods: Eightytwo subjects were recruited (44 female) with a mean age of 26.2 years. Both wrists were scanned on dorsal and volar aspects with diagnostic ultrasound. Age, gender, and the presence of wrist symptoms at the time of the scan were recorded. History of fracture or surgery to the wrist excluded subjects. Results: The prevalence was $50 \%$ (42 of 84 subjects). Twenty-nine ganglion cysts were found in the dominant hand compared to 23 found in the nondominant hand. Out of those, 40 were found on the dorsal aspect, and 12 were found on the volar aspect of the wrist. Thirty-seven percent of the students studied had pain as a symptom. Out of those, only $18 \%$ were positive for a ganglion. Conclusion: The prevalence of ganglion cysts in a chiropractic student population was similar to that reported in the general population. (This is a conference presentation abstract and not a full work that has been published.)

\section{Decrease of thyroid autoimmune activity after hypothalamic-pituitary-adrenal axis testing and anti-inflammatory polytherapy: A case report}

Christopher Arick

Objective: This case report illustrates a patient with chronic fatigue and inability to lose weight that presented to a chiropractic medicine clinic. The patient was treated using an anti-inflammatory polytherapy of dietary changes, lifestyle modifications, and targeted orthosupplementation after hypothalamic-pituitary-adrenal axis laboratory testing. Clinical Features: A 34year-old woman had complaints of fatigue and inability to lose weight for 2 years. She was found to have high serum thyroglobulin antibodies, low serum vitamin D3, low saliva dehydroepiandrosterone-sulfate, and an attenuated saliva cortisol pattern. Intervention and Outcome: The patient was placed on an ancestral diet and recommended changes in her exercise routine. She was also given a treatment plan of targeted supplementation per conventional and functional laboratory results. After 12 weeks, the patient reported increased energy and weight loss. Her thyroglobulin antibodies returned within reference range, salivary cortisol increased and followed proper circadian rhythm, and dehydroepiandrosterone-sulfate increased. Conclusion: This report describes improvement in a patient's thyroid autoimmunity through an anti-inflammatory polytherapy of dietary change, lifestyle modification, and supplementation specific to conventional and hypothalamic-pituitary-adrenal axis laboratory testing. This case illustrates that thyroglobulin antibodies can decrease to within reference range and correlate with the patient's symptomatic improvement. (This is a conference presentation abstract and not a full work that has been published.)

\section{Overcoming barriers to publication: A case report of a chiropractic researcher}

Barclay Bakkum, Robert Cooperstein, Cynthia Chapman

Objective: Only about one-third of projects submitted to chiropractic research conferences are ultimately published. The phenomenon of not following through on publishing articles based on studies presented at research meetings may be because of barriers that are both institutional and time management issues. Clinical Features: After hearing a presentation on publication rates at chiropractic research conferences, a chiropractic researcher recognized that the issue of not publishing articles based on presentations at meetings was affecting his productivity and causing him mental stress. He contacted the presenter to provide feedback 
on how greater awareness of this issue impacted him personally and to share insights on how to address this problem. Intervention and Outcome: After identifying several exacerbating factors, the presenter and researcher agreed that the chief barriers to publication in this instance were mostly time management issues. The subject is now submitting 1 paper per month after various behavioral modifications. Conclusion: Barriers to publication are amenable to resolution if contributing factors are addressed. Thought and dialogue may factor as much as or more than institutional changes. This case is an example of how 1 researcher translated investigational information on barriers to publication into actually breaking through those barriers. (This is a conference presentation abstract and not a full work that has been published.)

\section{Dynamic assessment of lateral ankle ligament instability and anterior impingement: A sonographic case report}

\section{Patrick Battaglia, Katherine Craig, Norman Kettner}

Background: Inversion ankle sprains are common and result in injury to the lateral ankle ligamentous complex. Recurrent ankle injuries cause instability resulting in functional deficits and increased risk for ankle osteoarthritis and subsequent impingement. Objective: The objective was to present a case report that utilized diagnostic ultrasonography (US) and radiography to document chronic injury to the lateral ligament complex, early osteoarthritis, and functional impingement. Methods: Case report of a patient with chronic ankle pain, instability, early osteoarthritis, and functional impingement. Results: US examination visualized a chronic full-thickness anterior talofibular ligament tear and subsequent osseous changes consistent with osteoarthritis. Radiographic examination was obtained to document the extent of ankle osteoarthritis. Anterior ankle impingement was also visualized under US. Discussion: US is useful when examining inversion ankle injuries, as it provides information regarding the integrity and functionality of the lateral ankle ligaments. When atypical osteoarthritis of the ankle is present, a history of repetitive inversion sprains should be considered, as chronic ligament damage may cause joint instability and subsequent degeneration. Conclusion: This case report exemplifies the utility of US in diagnosing ligamentous, articular, and osseous injuries of the ankle pertinent to making informed clinical decisions. (This is a conference presentation abstract and not a full work that has been published.)

\section{Case report: Resolution of cervicogenic cephalgia and greater occiptial neuralgia with collaborative care}

David BenEliyahu, Jacob Rauchwerger, Alan Kaell, Neha Naik, Michael Tofano

Background: A 42-year-old female with a chronic history of "migraine" headaches was previously treated by her primary care provider with Imitrex. She reported occipital and suboccipital headache as well as neck pain. An orthopedist referred for chiropractic care. Examination revealed cervical joint dysfunction, trigger points of the suboccipital, splenius, and semispinalis muscles. Deep tendon reflexes were normal, with hyperesthesia at $\mathrm{C} 2 / 3$. Her working diagnosis was cervicogenic cephalgia and occipital neuralgia. Objective: This case report demonstrates that interprofessional collaboration can help achieve successful resolution of a patient's problem. Methods/Results: The patient was treated with spinal manipulative therapy, myofascial release, ultrasound/galvanism, traction, and trigger point injections. Clinically significant relief was seen with $80 \%$ reduction in frequency and intensity of her headaches. Collaboration with an anesthesia pain specialist for greater occipital nerve blocks resulted in complete resolution of her headaches. Discussion: Headache conditions can have unrecognized multicomponent aspects that can lead to ineffective treatment and resolution. Collaborative care helps delineate an accurate diagnosis and successfully resolve the condition through combined interventions at all components. Conclusion: Interprofessional collaboration can help a patient achieve complete resolution of a complex problem with accurate diagnosis and integrated care. (This is a conference presentation abstract and not a full work that has been published.)

\section{Assessing the change in chiropractic student scores in a review course for the National Board of Chiropractic Examiners Part I examination provided as a required course by an accredited chiropractic college}

Judy Bhatti, Elissa Twist

Background: Many students take the National Board of Chiropractic Examiners (NBCE) Part I after completion of their 1st year in chiropractic college. Many students spend substantial resources preparing for this high-stakes exam, and many colleges develop programs to foster success on the exam. Objective: Investigate the mean class test score change and the perceptions of chiropractic students about their preparedness and confidence to pass the NBCE Part 1 examination after a life sciences review course. Methods: A test and survey used to assess student recall of life sciences material and perceived preparedness and confidence, respectively, were administered before and after a life sciences review class. Mean test score changes were computed, and frequencies were computed for survey answers. Results: Students experienced a 2.6-point mean increase from the pre- to posttest scores. The number of students feeling prepared and confident for national boards decreased after the life sciences review course. Discussion: This study has given the instructors valuable insight on what students desire in a review course. Conclusion: Students experienced an increase in their review class test scores and a slight increase in confidence in their ability to pass the NBCE exam. (This is a conference presentation abstract and not a full work that has been published.) 
Integration of sacro-occipital technique cranial therapy with an occlusal splint for the treatment of obstructive sleep apnea: A case report

Thomas Bloink, Charles Blum

Introduction: A 56-year-old female patient presented for chiropractic and dental care with persistent symptoms of sleep apnea, excessive daytime sleepiness, short-term memory loss, foggy-headedness, temperomanibular joint pain, chronic myofascial neck and shoulder pain, fatigue, and vertigo. Methods/Intervention: Cranialdental exam revealed dental class II, narrow arches, and premature anterior contacts with clenching and bruxism. The sleep study revealed a Respiratory Disturbance Index (RDI) of 17.1 and an Apnea Hypopnea Index (AHI) of 16.3, with the lowest oxyhemoglobin saturation $\left(\mathrm{SaO}_{2}\right)$ of $89 \%$ during sleep. Six treatments over a 3-4-week period of time consisted of sacro-occipital technique and cranial care with dental care to facilitate occlusal balancing. Results: Following the 6 office visits, the patient reported significant reduction of all symptoms. Follow-up polysomnogram was performed 1 month following the study and with the dental appliance in her mouth. Both RDI and AHI were reduced to 2.9, and the lowest $\mathrm{SaO}_{2}$ was $92 \%$ during sleep. Due to her increased ability to sleep and increased oxygenation, she had less daytime fatigue and greater function. Conclusion: The persistent nature of the patient's apnea, the pre- and postsleep study objective findings, and the patient's significant reduction in pain and improved function are compelling features of this case. (This is a conference presentation abstract and not a full work that has been published.)

\section{Improvement in clinical outcomes after dry needling in a patient with occipital neuralgia}

Bryan Bond, Christopher Kinslow

Background: Dry needling (DN) is defined as a procedure that involves inserting a dry needle into a myofascial trigger point (MTrP) with the goal of inactivating a trigger point and mitigating pain. Objective: The primary purpose of this case report is to outline the diagnosis, intervention, and clinical outcome of a patient presenting with occipital neuralgia. Methods: The patient described a 4-year history of stabbing neck pain and headaches. Examination findings included MTrPs in the bilateral upper trapezii muscles along with the right suboccipital muscles. After providing informed consent, the patient underwent a total of $4 \mathrm{DN}$ sessions over a 2-week duration. During each of the treatment sessions, needles were inserted into the trapezii and suboccipital muscles. Results: Following $4 \mathrm{DN}$ sessions, the patient reported a $32-$ point change in her neck disability index score along with a 28-point change in her headache disability index score. Discussion: Thus, it appears that following the subsequent 4 sessions of DN over 2 weeks, our patient experienced meaningful improvement in her neck pain and headaches. Conclusions: To the best of our knowledge, this is the 1st case report describing DN to successfully improve clinical outcomes in a patient diagnosed with occipital neuralgia. (This is a conference presentation abstract and not a full work that has been published.)

Intervention in menorrhagia through chiropractic adjustment and spondylotherapy: A case report

William J. Boro

Background: Since menorrhagia affects about 10 million American women yearly and the most common treatment is hysterectomy, investigation of a more conservative approach is in order. Objective: The purpose of this report is to describe the clinical course, treatment, and immediate response of a female patient suffering from uncontrolled uterine bleeding of over 2 weeks' duration to the application of chiropractic adjustments and the use of spondylotherapy. Methods: Treatments consisted of sacro-occipital technique category 2 block placement; adjustments to the thoracic, lumbar spine, and cranium; and spondylotherapy to C7, L3, and L5. Results: Patient reported that the bleeding stopped the day after the 1st treatment. She also reports that her periods have become more regular, with considerably reduced pain. Discussion: Dysmenorrhea is associated with an increase in uterine vasculature. I propose that one of the reasons for the successful treatment with spondylotherapy is its effect on returning tone to the uterine vasculature, thus reducing the blood flow and loss from the uterine lining both during the cycle and at menstruation. Conclusion: This case report discusses an approach, based on neurophysiology, as an alternative to the standard pharmaceutical and surgical approaches to treating menorrhagia. (This is a conference presentation abstract and not a full work that has been published.)

\section{The return of color vision secondary to macular degeneration after chiropractic care}

Thomas Brozovich

Objective: To discuss the chiropractic management of a patient whose unilateral color vision loss associated with macular degeneration resolved after treatment. Clinical Features: A 66-year-old female with a history of cervical, thoracic and lumbar pain and a 4-year history of macular degeneration of the left eye resulting in a loss of color vision sought chiropractic care primarily for her spinal pain. Initially, she was not requesting care for her loss of color vision. She reported having multiple recent traumas (falls) injuring her cervical, thoracic, and lumbar region. Intervention and Outcome: The patient was adjusted based on location of her subluxations (intersegmental joint dysfunction). She had 17 treatments over 8 months. A thermography study was performed of the face before and after treatment, and a more symmetrical thermal pattern was obtained. Her spinal pain reduced. She also unexpectedly had a return of color vision in her left eye. Conclusion: The patient responded favorably to chiropractic care, which resulted in a decrease in spinal pain and improvement in left-eye color vision. (This is a conference presentation abstract and not a full work that has been published.) 
The relationship between chiropractor required and current level of business knowledge

Michael Ciolfi

Background: Chiropractors frequently practice within health care systems requiring them to have the business acumen of an entrepreneur. Objective: The purpose of this quantitative correlational study was to examine the relationship between chiropractors' perceived level of business knowledge and perceived level of current business knowledge. The theories of reasoned action and planned behavior drove the theoretical framework of this study. Methods: Two hundred and seventy-four participants rated the level of perceived knowledge required and their current perceived level of knowledge for the same 8 items. Data were analyzed using Spearman ranked correlation to determine the statistically significant relationships for each variable. Results: The results of correlation testing signified a positive correlation for knowledge required and current knowledge for 6 variables: (1) organizational behavior, (2) strategic management, (3) marketing, (4) legal and ethical, (5) managerial decisions, and (6) operations. Discussion: The findings indicate that the curricula of chiropractic colleges might require change in their course offerings to include a greater amount of business education. Conclusion: The implications of this study for social change include the potential to improve chiropractors' business knowledge and skills, enabling practice success and enhanced health services delivery toward sustainable health care goals. (This is a conference presentation abstract and not a full work that has been published.)

\section{Comparison of 4 variations of the manual muscle} test of the sartorius

\section{Katharine Conable, Tracy Edelmann}

Background: The manual muscle test (MMT) of the sartorius is described and illustrated differently in different MMT references. Four variations were identified based on the tester's hand placement. Objective: To identify the MMT variation that best isolates the sartorius from the medial hamstrings. Methods: With insitutional review board approval, 23 volunteer subjects (18 male, 5 female) between 20 and 58 years old were tested on 4 variations of the sartorius MMT. Each test was repeated twice, with a 10 -second rest between tests. Measures: Force at the lower testing hand and surface electromyographic (sEMG) amplitude from the right sartorius and medial hamstring. Results: Ratio of root mean square of sEMG amplitude between the sartorius and medial hamstrings was compared with a repeated measures analysis of variance. Test variants differed with $\mathrm{p}<0.0001$ overall. Variant $\mathrm{C}$ differed from all others, $\mathrm{p}<0.001$, with the sartorius-to-hamstring ratio higher than the other tests. Test C used lower force than other tests. Discussion: Variant $\mathrm{C}$ has not been previously published. Generalizability of this study is limited by the use of a single tester. Conclusion: MMT of the sartorius with a flathand lower contact appears to minimize confounding contribution of the medial hamstrings. (This is a conference presentation abstract and not a full work that has been published.)

The effect of chiropractic care in a patient with menorrhagia, metrorrhagia, dysmenorrhea, and irregular menstrual cycle length

Karima Cooper, Linda Mullin

Objective: This case highlights the outcomes of chiropractic spinal correction on concomitant reproductive issues in a 38-year-old female. Clinical Features: The patient presented to a chiropractic office for myriad symptoms that began 4 years earlier after the birth of her 1st child. Along with neck and low back pain, her most distressing symptoms included menorrhagia (prolonged menstrual bleeding), metrorrhagia (vaginal bleeding between menses), dysmenorrhea (painful menses), and irregular cycle length. Her menses frequently lasted more than 2 weeks with breakthrough bleeding between cycles. Even with use of the birth control pill, symptoms persisted. She was also diagnosed with uterine fibroids. Intervention and Outcomes: She was treated with high-velocity, low-amplitude adjustments on a drop table along with spinal traction, wobble board, and head and hip weighting. Resolution of back and neck pain was accompanied by ultrasoundconfirmed shrinkage of uterine fibroids and all associated menstrual symptoms. There was a return of normal cycle length without the use of the birth control pill. Conclusion: This case demonstrates the resolution of concomitant reproductive symptoms associated with vertebral subluxation. (This is a conference presentation abstract and not a full work that has been published.)

\section{Intra- and interobserver reliability of the Cobb measurement by chiropractic interns using digital evaluation methods}

Jesse Cracknell, John Taylor, Doug Lawson

Introduction: It is important to create a body of evidence surrounding the reliability of certain diagnostic criteria. While the reliability of the Cobb measurement is well established with various licensed health care professionals, this study aims to determine the intra- and interobserver reliability of the Cobb measurement among chiropractic interns. Methods: Fourteen chiropractic interns analyzed 10 preselected digital spinal radiographs on a picture archiving and communication system in 2 separate rounds of observation. The participants indicated their choice of end vertebra and Cobb measurement in each round of observation. Agreement on vertebral levels selected was estimated using percentage agreement. Intraobserver reliability was estimated using the Pearson $r$ correlation coefficient, and interobserver correlation was estimated using the interclass coefficient (ICC). Results: The range of percentage agreement on vertebral level selection was 0.36-0.79. The Pearson $r$ correlation coefficient for rounds 1 and 2 was 0.79 . The $\operatorname{ICC}(3,1)$ was 0.79 (round 1) and 0.70 (round 2). Conclusion: Less-than-optimal agreement on end vertebrae selection was found 
between observers. Intra- and interobserver reliability of the Cobb measurement was "excellent" (round 1) and "good" (round 2). (This is a conference presentation abstract and not a full work that has been published.)

\section{The influence of the chiropractic treatment in patients with insulin resistance associated with diabetes type 2: A case series}

Fabio Dal Bello, Patricia Bergesch, Charles Blum

Introduction: High plasma levels of insulin and glucose due to insulin resistance are a major component of the metabolic syndrome, a syndrome with a group of risk factors that raises the risk for heart disease and other conditions, such as diabetes mellitus (DM) type 2 and stroke. Since some low-level evidence has found that chiropractic care may demonstrate some influence in nonmusculoskeletal interactions, such as in DM individuals, this study was performed to analyze any possible influence of chiropractic treatment for patients with DM and insulin resistance. Methods: Four patients with DM type 2 with insulin resistance were selected by an allopathic diabetes specialist to participate in this study. The treatment consisted of 8 chiropractic office visits that incorporated adjustments to the spine and chiropractic manipulative reflex technique to balance viscerosomatic/somatovisceral autonomic reflexes. Laboratory blood tests were taken on the 2nd and 8th office visits, before and after the chiropractic adjustment. Results: After the 8 visits, the levels of insulin resistance, following chiropractic care, decreased significantly when compared to prior to the chiropractic intervention. Conclusion: Further research is needed in this arena to determine which subset of patients and type of chiropractic care would achieve the optimal results. (This is a conference presentation abstract and not a full work that has been published.)

\section{Development of a grand rounds course}

Renee DeVries, Louise Delagran

Background: Grand rounds have been common in medicine and medical education for over 100 years, though they have not historically been a part of the culture of chiropractic education. Objective: The purpose of this study is to describe the development of a grand rounds course for chiropractic students. Methods: Course development followed the ADDIE method of curricular design, a systematic approach using a process of analysis, design, development, implementation, and evaluation. Results: The grand rounds course is delivered 1 hour per week for 13 weeks. Instructional strategies include a brief lecture, case presentation by a faculty member, an online forum, and group presentations by students. Learning objectives and assessments allow demonstration of student acquisition of Council on Chiropractic Education metacompetencies, including the use of research evidence in clinical decision making. Discussion: As a course, grand rounds can be used as a way to familiarize future doctors with the profession's expectations for evidenceinformed patient care and the etiquette of professional exchange. Conclusion: This course serves the needs of our future doctors by exposing them to unique cases and enhancing their skills in evidence-informed practice. Additionally, it demonstrates students' attainment of important clinical skills for programmatic accreditors. (This is a conference presentation abstract and not a full work that has been published.)

\section{Evaluating chiropractic college mission statements: A model for assessment}

Scott Donaldson

Objective: This study considers the mission statements of a US chiropractic college relative to the strategic plan, goals, and actions. It also looks to establish a model for assessment of mission statements as an important part of the institutional assessment strategy. Methods: The mission statements and strategic plan were assessed based on an analysis of content, communication capacity, and applicability to the goals and actions of the college. Analysis included document review, a Fog Index for readability, and a connotative analysis questionnaire. Results: Most of the components existed within the mission statements. Any components that were not clearly communicated in the mission statements were clarified and made complete within the companion strategic plan and its goals. Most respondents agree to what the plan communicates, but newer employees are less sure. Conclusion: The mission statements contain most of the components searched for and communicate at a level that requires at least a college education and/or familiarity with chiropractic. Long-term employees and administrators understand the mission statements, and new employees seem to have some trouble with a few components as they relate to planning. The actions of the college are in alignment with the mission. (This is a conference presentation abstract and not a full work that has been published.)

\section{Case study: Post-sinus surgery insomnia}

Eva Elsangak

Introduction: No cases of complete insomnia following sinus surgery were found in the literature, only sporadic cases of sleep disturbances after craniotomy, kidney transplant, and heart surgeries. Case Presentation: A 25-year-old female with no history of sleep disturbance presented with total insomnia immediately after endoscopic sinus surgery, also complaining of constant neck, head, shoulder, low back pain, and midback tightness. Intervention and Outcome: Chiropractic care utilizing Activator Methods provided an immediate increase in ease of breathing, relaxed body, and ability to rest. Mental imaging of swimming relaxed the mind. In 2 weeks, the patient slept 60-90 minutes per night. At 4 weeks, the patient slept 2-3 hours and reported 3-5 hours per night of interrupted but restful sleep after 10 weeks of care. Discussion: Stress, prolonged anesthesia, and surgical cranial insult caused cranial, spinal, and rib subluxations. Correction of subluxations facilitated improved breathing, overall function of body, and sleep. Conclusion: The lack of literature on acute 
postoperative insomnia calls for more investigation and reports pertaining espceially to the efficacy of chiropractic care. (This is a conference presentation abstract and not a full work that has been published.)

\section{Tricipital supernumerary head of the biceps brachii muscle: An anatomical case study and clincial implications}

Dennis Enix, Kasey Sudkamp, Robbyn Keating, Anthony Aamodt, Frank Scali

Background: Nerve entrapments of the upper extremity are common occurrences and may be associated with anatomical variations in musculature. Background: This study presents an anatomical variation of the biceps brachii muscle that may have clinical significance. Methods: A gross dissection of a formalin embalmed cadaveric specimen was performed at a university anatomical laboratory. Results: A tricipital supernumerary head of the biceps brachii muscle was noted on 1 extremity in an embalmed human cadaveric specimen. Discussion: Anatomical variations of the biceps brachii muscle are found in $8 \%-38 \%$ of individuals, depending on the populations. Peripheral nerve entrapments may result from anatomical variations of the anterior compartment of the arm. Variations in the musculocutaneous nerve are associated with supernumerary heads of the biceps brachii. Conclusions: The presence of a 3headed biceps muscle may cause neurovascular compression of the median nerve, musculocutaneous nerve, or brachial artery, resulting in changes in strength and sensation. The patient who is refractory to care may warrant careful evaluation of the anterior compartment of the arm for potential musculature variations. (This is a conference presentation abstract and not a full work that has been published.)

\section{Improvements in quality of life in a 4-year-old male with cerebral palsy undergoing chiropractic functional neurology treatment: A case study}

Susan Esposito, Kaitlin Townsend

Objective: The purpose of this study is to highlight the improvements following chiropractic functional neurology treatment of a young male patient with acquired cerebral palsy following an ischemic brain injury. Clinical Features: A 4-year-old male presented to the clinic with cerebral palsy symptoms following an ischemic brain injury. The clinical impression was hypoxic ischemic encephalopathy secondary to acute trauma presenting as spastic, quadriplegic cerebral palsy. Intervention and Outcomes: Intervention included multiple sessions of vestibular stimulation therapy on a whole-body off-axis rotational device, which is a multiaxial, computer-operated rotational chair. Strategies also included vibrational stimulation, complex body movements, optokinetic exercises, and chiropractic adjustments. Improvement in quality of life and activities of daily living resulted. Conclusion: This case suggests the use of novel chiropractic treatment strategies in the management of severe neurologic dysfunction, such as cerebral palsy. Further research into treatment of this type for patients with cerebral palsy is warranted. (This is a conference presentation abstract and not a full work that has been published.)

\section{An investigation into the efficacy of fear- avoidance beliefs as a predictor of treatment outcomes in McTimoney chiropractic patients \\ Carole Filby, Gabrielle Swait, Adrian Hunnisett, Christina Cunliffe}

Introduction: Fear-avoidance beliefs (FABs) have been studied extensively in relation to patients with low back pain, but less is known about their relevance to patients presenting with other types of musculoskeletal pain. This study sought to establish whether a link existed between FABs and treatment outcomes in patients undergoing chiropractic treatment. Method: Following ethical approval, an interventional prospective cohort study of 46 new patients presenting for chiropractic was undertaken. Patients answered questions capturing their FAB levels, pain, and disability prior to their 1st treatment and then again after 4 treatments. Data were analyzed to establish any relationship between FAB levels and treatment outcomes. Results: Patients showed a significant $(p<.0001)$ average improvement of 4.94 points on the Roland-Morris Disability Questionnaire following chiropractic care. Patients with high levels of FABs made an average of a 3.885-point improvement following treatment, while patients with low FABs improved by an average of 6.00 points $(p<$ .05 ). Conclusion: FABs may be an accurate predictor of treatment outcomes in chiropractic patients. In addition to strengthening the evidence base for the efficacy of chiropractic, this study endorses the need for practitioners to be aware of FABs and strategies for combatting them. (This is a conference presentation abstract and not a full work that has been published.)

\section{A cross-sectional study to determine current basic public health markers of faculty clinicians at a chiropractic teaching institution}

Rod Floyd, Kenice Morehouse, Stephen Grand, Shane Carter, Craig Mencl

Introduction: As the wellness program progresses at this chiropractic teaching institution, we wanted to check our faculty clinicians' knowledge of their basic public health markers. Methods: Faculty clinicians were surveyed $(=14)$. A questionnaire was used to gather data regarding body mass index (BMI) scores, waist circumference, blood pressure, activity level, tobacco use, and consumption of alcohol, along with vegetable and fruit intake. Results: Females outperformed the males in 5 of the 8 categories, including BMI, blood pressure, exercise, alcohol consumption, and fruit consumption. The males outperformed the females in waist circumference and vegetable consumption, and the males and females tied in tobacco use because none of the clinicians use tobacco products. Discussion: Many of the studies that have been published have focused on students and patients. This study focused mainly on faculty clinicians at a chiropractic teaching institution. It is important that doctors of chiropractic, whether in teaching institutions or in private practice, 
model health behaviors that will inspire students and patients to become healthier and improve outcomes. (This is a conference presentation abstract and not a full work that has been published.)

\section{Chiropractic and dental care of a patient with temporomandibular and sacroiliac joint hypermobility: A case report}

Richard Gerardo, Charles Blum, David Shirazi

Introduction: The purpose of this case report is to share a novel protocol for the treatment of chiropractic and dental treatment of a patient with temperomandibular disorder who presented with hypermobility of the sacroiliac joint (sacro-occipital technique category 2) and temperomandibular joint disorder (TMJ). Case History: A 47-year-old female patient presented with chief complaints of pain when chewing, jaw pain, limited mouth opening, and TMJ crepitus. Following dental evaluation and delivery of dental orthotics, the patient was referred for concurrent chiropractic, which found indicators of a category 2. Methods/Intervention: Following orthotic and chiropractic care at 8 weeks, the capsulitis of TMJ was no longer present, and procaine injections were initiated at 1-month intervals, followed by prolotherapy. Chiropractic and dental care was continued over a 10-month period. Results: Following concluding treatment, the patient is free of most pain, has full range of motion in the cervical and lumbar spine and negative sacroiliac hypermobility findings, and can open her mouth greater than $42 \mathrm{~mm}$ (initially presented with a 28-mm opening) with normal joint tracking and translation. Conclusion: Concurrently, her sacroiliac joint hypermobility syndrome and related soft tissue tension patterns in the lower extremity, cervical spine, and jaw region have also significantly improved. (This is a conference presentation abstract and not a full work that has been published.)

\section{Chiropractic intern attitudes, beliefs, and intentions with regard to health promotion, wellness, and preventive services: Phase 2}

Stephen Grand, Kenice Morehouse-Grand, Shane Carter, Rod Floyd

Background: Phase 1 results demonstrated both a need and a willingness by interns to learn more about wellness. This is supported by various stakeholders. Objective: Determine the effectiveness of a wellness training in a chiropractic clinic. Methods: The improved beta-test model for wellness practice introduction, previously utilized, was employed to instruct all clinic interns. The full class of interns was surveyed before and after a program of educational interventions. Results: Interns felt that wellness was a part of chiropractic care but felt lacking in preparation for it. The intervention had mixed results in terms of educational outcomes. Discussion: Although interns generally appreciated wellness, presurvey comments also suggested disappointment in their current relevant education. Despite only modest changes in the postsurvey, comments were favorable. The mixed response may have been due to the incomplete training and buy- in by their mentor-clinicians. Conclusion: Limitations include a serious loss to follow-up and a bias because beta-test mentors were wellness practitioners, but other mentors had different styles. The infrastructure in the clinic is slowly developing, and this should enhance future efforts. Further development is indicated. (This is a conference presentation abstract and not a full work that has been published.)

\section{Improving a patient's quality of life after a traumatizing fall causes structural impairments and disability: A case report illustrating collaborative health care benefits}

Joseph Haezebrouck

Objective: To discuss chiropractic care of a patient focused on recovery from life-threatening injury. Strategic care engages support of specialized providers to improve the patient's functional ability vs disability. Clinical Features: A nonambulatory 61-year-old female presented for chiropractic care after hospitalization from falling down icy stairs. She was diagnosed with fractures of her right tibia, fibula, and talus, and extensive surgical repair, implanted arthrodesis, infection, diabetic complication, and Clostridium difficile colitis. Isolation recovery for 20 days was halted by a loss of insurance coverage. The patient was released as "disabled" by a physician. Intervention and Outcome: The patient was referred to a university clinic. Consultation, care plan, patient consent, and institutional review board exemption forms were signed. Chiropractic services, radiology, exercise programs, gait training, stabilizing custom orthotics, and resistive strengthening were correlated and implemented. The patient's commitment compliance was focused with the intent for recovery. The patient's activities of daily living were regained, disability status was lifted, and minimal impairment of the right ankle exists. Quality of life showed marked improvement. Conclusion: Collaborative care management, quantifying outcomes, and recording patient assessments will always valuate chiropractic care. (This is a conference presentation abstract and not a full work that has been published.)

\section{Resting pulse rates following adjustment of C1 subluxation: A case series}

John Hart

Background: Resting heart (pulse) rate (PR) is an autonomic marker that may be useful in subluxationcentered chiropractic practice. Objective: The present study investigated the change in PR following chiropractic adjustment of $\mathrm{Cl}$ subluxation. Methods: A convenience sample of 23 chiropractic students was examined on 3 visits: 2 preadjustment visits and 1 postadjustment visit. The outcome variable was the difference in PR between the 2nd preintervention visit and the postintervention visit using the paired $t$ test. Results: A decrease in postintervention PR was observed for the group but was not statistically significant (mean reduction of 2.7 beats per minute $[\mathrm{BPM}], p=.243)$. A statistically significant reduction in PR was observed, with a large effect size for males $(n=$ 
12; mean reduction $=8.0 \mathrm{BPM}, p=.014$, effect size $=$ 0.74). Discussion: Males and females may respond differently to clinical interventions, particularly in regard to cardiovascular function, which may account for the difference of findings between genders in the present study. Conclusion: In this preliminary study, a decrease in pulse rate was observed following chiropractic adjustment for atlas subluxation but was statistically significant only for males. Further research with other chiropractic techniques may show different results. (This is a conference presentation abstract and not a full work that has been published.)

\section{Resolution of low back pain in an 8-year-old patient following upper cervical chiropractic care: A case study}

Charmaine Herman

Background: The prevalence of low back pain (LBP) in children has been estimated at $50 \%$ beginning as early as 4 years of age. Chiropractic management, inclusive of specific upper cervical (UC) chiropractic management, may also provide successful outcomes for children with LBP. Objective: To review the effectiveness of using a specific UC chiropractic technique in the case of an 8-year-old female patient with LBP. Methods: This is a retrospective case report detailing the 5-month care plan of a pediatric LBP patient under a specific technique protocol to correct an UC vertebral subluxation. Results: An 8-year-old patient reported resolution of LBP following Blair UC chiropractic care. Discussion: The Blair UC chiropractic technique analyzes the relationship between the occipital condyles and atlas lateral mass articulations to identify vertebral subluxations. Using specific radiographs and adjustments, the reduction of the UC subluxation may result in reduction of compensatory subluxations throughout the spine. Conclusion: Based on the significant and timely improvement of this patient, this case report demonstrates the need for further investigation into the use of a noninvasive, light-force, cost-effective, and highly specific method of correcting UC subluxations, which have an effect on this condition and potentially similar cases. (This is a conference presentation abstract and not a full work that has been published.)

\section{Changes in state regulation pertaining to chiropractic staff}

\section{LaKeisha Holloway, Catherine Eberhart, Stacie Martel}

Background: The purpose of this study is to explore the existence and extent of increases in state chiropractic board regulation regarding chiropractic staff for the period from 1995 through 2014. Changes in such requirements potentially impact curriculum in chiropractic staff education or training programs. Methods: Following institutional review board exemption, questionnaires were sent to representatives of each chiropractic licensure board requesting specified information about current and past requirements impacting chiropractic supportive personnel and plans for future regulation. Similarly, current and past data were also sought via each chiropractic board. Findings were compared to formerly published peer-reviewed research and were then categorized into 3 primary categories and 1 subcategory. Results: Findings were that, first, $6 \%$ of chiropractic boards indicated via questionnaire that they are considering statutory or regulatory change impacting chiropractic staff in the next 5 years. Second, $57 \%$ of chiropractic boards were determined to have enacted at least minimal increases in regulation regarding chiropractic staff. Within that category, an $18 \%$ increase specifically related to physiotherapy was noted. Third, $43 \%$ of boards were determined to have no change related to chiropractic staff. Conclusion: Chiropractic state board requirements have increased over the past 20 years, particularly related to delegation and physiotherapy. (This is a conference presentation abstract and not a full work that has been published.)

The feasibility of using electronic health records as a data source for practice-based research: An exercise in interprofessional collaboration

Ronald Hosek, Stephanie Sullivan, Edward Owens, Joe Esposito

Background: While the potential value of practice-based research networks (PBRNs) is generally acknowledged, chiropractic PBRNs have been difficult to establish and sustain. Objective: To develop a PBRN with a cloudbased electronic health record (EHR) as its data source. Methods: A collaborative project was undertaken to develop a PBRN around a cloud-based EHR system. An implementation plan was created and institutional review board approval obtained. In accordance with the plan, recruitment of clinics was undertaken, data handling and quality control protocols were developed, and data were extracted from the database. Results: Twenty-one clinics agreed to participate. Seventeen of the variables in the database were selected for a snapshot of a month's worth of retrospective data. The query yielded more than 30,000 records, of which 20,000 were uaable. Discussion: A cloud-based PBRN appears to have the same challenges as distributed networks (eg, recruitment, data quality control, and the trade-off between identity protection and data integrity). Conclusions: Our experience suggests that a cloudbased EHR system offers great potential for providing larger pooled data sets with relatively greater ease than traditional PBRNs. (This is a conference presentation abstract and not a full work that has been published.)

\section{Cervical herniated disc with cervical radiculopathy symptoms improved under conservative chiropractic care: A case report}

\section{Todd Hubbard, Brian Hall, Janice Hubbard}

Objective: There are relatively few papers discussing cervical disc herniation $(\mathrm{CDH})$ and cervical radiculopathy (CR) treated conservatively in the literature. This is a case report of a 58-year-old male with $\mathrm{CR}$ and $\mathrm{CDH}$ who experienced a resolution of his symptoms after cervical spinal manipulative therapy and postural taping. Clinical Features: This patient was experiencing bilateral $\mathrm{CR}$ and 3 sessions of magnetic resonance imaging confirmed $\mathrm{CDH}$ after a motor vehicle accident. 
After 4 weeks of chiropractic treatment produced no improvement and the patient was told he was not a candidate for surgery, a 2nd round of chiropractic treatment was a success. Intervention and Outcome: After the 1st course of chiropractic care failed to produce results, the treatment was reevaluated. The patient then received a combination of Blair upper cervical adjustments to the upper cervical spine, passive segmental range of motion to the midcervical spine, and high-velocity, low-amplitude rotational adjusting to the cervical-thoracic junction and postural taping. Conclusion: Symptoms resolved after receiving a combination of conservative chiropractic treatment. Future research on how combinations of chiropractic treatments help the $\mathrm{CDH}$ patient should be pursued. (This is a conference presentation abstract and not a full work that has been published.)

\section{Implementation of a chiropractic care program for soldiers, veterans, and their families in a chiropractic college clinic system}

Julie Johnson, Shayan Sheybani, Ron Boesch

Background: Addressing the health care needs of today's soldier requires recognition of dramatic changes to military service over the past 25 years. Musculoskeletal health has a significant impact on each soldier's ability to perform. Chiropractic care is a conservative approach that can benefit this population. Objective: To demonstrate implementation of a military care program (MCP) in a chiropractic college clinic system. Methods: The chiropractic college clinic system initiated the MCP. It was established to provide chiropractic services for active-duty soldiers, dependents, and military veterans. Results: Current MCP results revealed a number of critical factors to be addressed in a chiropractic clinical care program of this nature. With 2,500 military visits in fiscal year 2008-2009, the MCP has grown to offer over 12,000 visits college-wide for fiscal year 2013-2014. Discussion: The focus of a chiropractic education program is graduation of a competent clinician. The experience of complex case presentations, through care of the modern soldier, affords the opportunity to challenge a chiropractic student to think beyond routine care. Conclusion: The increased volume of services provided is 1 metric to determine success but could also be explained as a result of close proximity to a military installation. (This is a conference presentation abstract and not a full work that has been published.)

\section{A preliminary investigation into the effects of cervical spinal manipulation on cardiac autonomic control by measuring heart rate variability}

Antonia Kam, Adrian Hunnisett, Christina Cunliffe

Introduction: The aim of this study is to determine whether upper cervical adjustment of $\mathrm{Cl}$ elicits a response from the cardiac autonomic control system in terms of producing a predominant parasympathetic or sympathetic response posttreatment in contrast to the response of the individuals undergoing sham and control groups. Method: Following ethical approval, a single-blinded randomized controlled trial was undertaken. Subjects were randomly assigned to 3 groups: intervention (upper cervical adjustment of $\mathrm{C} 1$ ), sham protocol, or control. The heart rate variability (HRV) data were recorded and analyzed over the 5-minute pretreatment and posttreatment intervals. Results: In asymptomatic adults, cervical spinal adjustment was associated with changes in HRV that were not seen with the sham procedure. In the intervention group, there were significant increases in aspects of $\operatorname{HRV}(p<.05)$. In subjects undergoing sham adjustment, there was no statistically significant change in the HRV measurements. Conclusion: An upper cervical spinal adjustment to $\mathrm{C} 1$ appears to influence autonomic cardiac control in ways that are not duplicated by a sham procedure or control. The HRV results for the intervention group show a significant increase in overall sympathetic output. (This is a conference presentation abstract and not a full work that has been published.)

\section{Clinical and ultrasound evaluation of an angioleiomyoma in the lower extremity: A case report}

Brooke Van Kirk, Aaron Welk, Aimee Jokerst, Norman Kettner

Objective: The purpose of this case study is to demonstrate the role of sonographic examination in the initial evaluation of an angioleiomyoma and to discuss the characteristic findings associated with this soft tissue mass. Clinical Features: We report a case of a 52-year-old female who presented with a large, tender, erythematous mass on the anterolateral aspect of her right knee. Sonographic examination revealed a highly vascular mass within the subcutaneous tissues. Differential considerations included benign soft tissues masses, such as angioleiomyoma and hemangioma. Intervention and Outcomes: Surgical consultation was recommended. Excisional biopsy was performed. Histolopathological examination confirmed the diagnosis of angioleiomyoma. Conclusion: Although ultrasound findings of a superficial soft tissue mass may be nonspecific, when a highly vascular, well-defined, slow-growing mass is present, angioleiomyoma should be included in the differential diagnosis. (This is a conference presentation abstract and not a full work that has been published.)

\section{Students' perceived confidence in specific elements of the physical examination 1 year posttraining: A survey and focus groups of 3 student cohorts}

\section{Lisa Killinger}

Background: In chiropractic education, physical examination (PE) training may be completed 1 year before students' PE performance on outpatients. Aims: To identify elements of the physical examination in which student interns feel least confident and guide curricular response to enhance areas of perceived weakness. Methods: One year after PE training, 3 cohorts of student interns were surveyed to determine which PE elements they felt the least confident in. Three cohorts of students $(n=256)$ were assessed by written 
questionnaire and follow-up focus group to gain more detailed information. Results: Results were pooled and reported as a combined cohort due to homogeneity of results. Of the 256 respondents, $53 \%$ were the least confident about the heart exam, and $24 \%$ listed the lung exam. The next most frequent responses were eye, neurological, and cognitive exam. Other responses were significantly less common. In all 3 cohorts, heart exam had twice the responses as lung exam. Focus groups unearthed that interpretation of findings and patient management were more challenging to students than the performance of the PE itself. (This is a conference presentation abstract and not a full work that has been published.)

\section{Dorsal scapular artery: Anomaly of rare origin \\ Kelsey Martin, Everett Johnson, Bahram Sardarabadi}

The dorsal scapular artery (DSA), also known as descending scapular artery, is a blood vessel that arises from either the 2 nd or the 3 rd part of the subclavian artery in approximately $70 \%$ of the population. This study is the result of identification of a variation of the normal anatomy that was discovered during routine dissection of the neck at a chiropractic institution. On inspection of the 1st part of the subclavian artery on the left side, it was noted that there were 4 branches emerging from the thyrocervical trunk instead of the normal 3. The DSA arose from the thyrocervical trunk in this case and traversed the inferior and lateral aspect of the neck. At the point of its intersection with the brachial plexus, the DSA takes a course deep to the superior trunk of the brachial plexus. During the course of the DSA, it gives rise to several branches that appear to be supplying the paravertebral muscles, superior portion of the serratus anterior muscle, levator scapulae, and the rhomboid muscles. Origination of the DSA directly from the thyrocervical trunk has been previously reported in approximately $0.6 \%$ of the population. (This is a conference presentation abstract and not a full work that has been published.)

\section{Sonographic diagnosis of an acute Stener lesion: A case report}

Ross Mattox, Aaron Welk, Mero Jr. Nunez, Norman Kettner

Objective: The purpose of this case report is to describe the use of diagnostic ultrasound (US) in the diagnosis of a torn and displaced ulnar collateral ligament (UCL) of the thumb (Stener lesion) presenting to a chiropractic teaching clinic. Clinical Features: A 48-year-old female presented after a fall on an outstretched hand. She suffered hyperabduction and hyperextension of the left thumb. Intervention and Outcomes: Radiography demonstrated an avulsion fracture of the base of the 1st proximal phalanx. US revealed a torn UCL of the thumb that was displaced proximal to the adductor aponeurosis, making it a Stener lesion. The Stener lesion has poor outcomes with nonsurgical treatment. Surgical repair followed the diagnosis. Conclusion: US provided an accurate diagnosis without the need for more costly diagnostic work-up. Optimal outcome was achieved after an early diagnosis with US. (This is a conference presentation abstract and not a full work that has been published.)

\section{An unusual forearm deformity: Implications for chiropractic management}

Celia Maguire

Objective: Hereditary multiple exostoses (HME) is a bone dysplasia with a reported association with Madelung's deformity. This case report describes the presentation of a 5-year-old girl with HME and forearm deformity and discusses the difference between this and Madelung's. Clinical Features: A 5-year-old girl with a family history of hereditary multiple exostoses presented to a chiropractic college clinic. Radiographic analysis and physical examination of the elbow were performed at initial presentation and at 2year follow-up. Varus carrying angle of the elbow and lateral misalignment of the radius on the capitellum were apparent. Limited pronation was accompanied by elbow laxity. Intervention and Outcome: Chiropractic manipulation of the elbow was not indicated; the patient was referred to a pediatric orthopedic specialist for evaluation and management. The right arm was surgically repaired. At 2-year follow-up, the radial head was centered under the capitellum, and laxity was reduced. Pronation increased from 5 to 15 degrees. Conclusion: While hereditary multiple exostoses do not represent a contraindication to chiropractic manipulative therapy, it is important for the chiropractor to be familiar with the forearm deformities and predisposition to radial head dislocation that are associated with the dysplasia. (This is a conference presentation abstract and not a full work that has been published.)

\section{Effect of the Activator Methods technique on lowering hemoglobin a1c levels in type II diabetics: A pilot study}

Katrina Mayes, Anquonette Stiles, Larry Hansen, Timothy Guest, John Markham, Gwendolyn Tutt

Background: Type II diabetes is a major public health concern, as it accounts for $90 \%-95 \%$ of all diabetes cases. It is alarming, as it can be prevented through exercise, weight loss, and healthy dieting. Objective: To investigate the effectiveness of utilizing the Activator Methods technique on type II diabetics through monitoring hemoglobin A1c levels. Methods: Participants enrolled in the 6-month study on confirmation of type II diabetes diagnosis. Participants received adjustments following the Activator Methods technique and performed hemoglobin Alc testing at baseline and at 3 and 6 months. Results: There were 4 participants. The average A1c at baseline was $8.75 \%, 7.6 \%$ at 3 months, and $7.4 \%$ at 6 -month study closure. Each participant showed a decrease in Alc levels over the course of the study with the exception of 1 participant. Discussion: This pilot study shows a decrease in the A1c levels that may indicate improved long-term blood glucose control. Such an effect may directly reduce the risk of microvascular complications. Conclusion: A larger-scale study may demonstrate that correction of vertebral subluxation improves the body's response to insulin. 
(This is a conference presentation abstract and not a full work that has been published.)

\section{Where is the pain source? A patient with low back pain and bilateral avascular necrosis of the hips}

Amy Minkalis, Robert Vining

Objective: Low back pain (LBP) is a common symptom arising from many possible sources, creating a complex diagnostic picture. One example, remote pathology in the hip, can contribute to or cause LBP. Clinical Features: A 37-year-old male presented for an eligibility exam for an ethics-approved clinical trial studying LBP. He reported right-sided LBP and right hip pain lasting approximately 6-12 months. Symptoms were aggravated by weight-bearing activities. An evidence-based diagnostic examination revealed little indication for a primary pain source originating from low back structures. Radiographs revealed bilateral avascular necrosis (AVN) with evidence of left femoral head collapse. Intervention and Outcome: The patient was referred to a general practitioner and orthopedic surgeon to determine etiology and for surgical consultation. Bilateral total hip arthroplasty was recommended. Conclusion: Hip AVN contributed to an atypical presentation of LBP due to aberrant localization of pain and/or combined with altered biomechanical loading of musculoskeletal structures. Conservative treatment should be attempted in early-stage AVN to prevent bony collapse. This case describes AVN atypically presenting as LBP. An early AVN diagnosis represents an opportunity for interprofessional collaboration due to the availability of conservative treatment options that can help manage pain and preserve hip joint integrity. (This is a conference presentation abstract and not a full work that has been published.)

\section{Restoration of symmetrical muscle tone and infant reflexes after chiropractic intervention of a neonate with concomitant apneic episodes}

Linda Mullin, Haruka Yamazaki

Objective: This case study highlights the use of chiropractic adjustments of the spine of a neonate who displayed an incoordination of the nervous system demonstrated by asymmetrical muscle tone and primitive reflexes with concomitant apneic episodes. Clinical Features: A male neonate experienced recurrent apnea and cyanosis in the neonatal intensive care unit and presented to a chiropractic office at 12 days old for assessment and care. Weak and asymmetrical muscle tones and asymmetrical primitive reflexes were observed at his 1 st visit. Restricted head rotation to the right with dystonic neck muscles on the left upper cervical region were present. Intervention and Outcome: A light-force side-lying adjustment was applied to the atlas for 3 visits utilizing the Gonstead chiropractic technique criteria. Conclusions: Complete resolution of asymmetrical muscle tone and asymmetrical primitive reflexes were attained. There was no recurrence in apneic events. (This is a conference presentation abstract and not a full work that has been published.)
Complete resolution of mild idiopathic scoliosis in a 9-year-old female: Results from the dynamic and corrective SpineCor bracing system

Paul Oakley

Background: SpineCor bracing is a unique form of 3dimensional dynamic bracing for the treatment of idiopathic scoliosis. It incorporates a "corrective movement," essentially a unique reversal (combination of axial derotation, lateral shifting, and/or lateral bending) of the postural and spinal asymmetry. Clinical Features: On April 24, 2014, a 9-year-old female with scoliosis came with parents for consultation. On examination of the AP thoraco-lumbar view, it was determined that she had a mild 14-degree right convex thoracic curvature as measured by the Cobb method from TL1. Intervention and Outcome: Due to her age, the parents elected to brace their child. She was determined to be a "thoracic type 1" SpineCor classification and was braced accordingly. The bracing had an immediate effect of overcorrecting her spine into a left thoracic curve. Close management was pursued to not lead to overcorrection. At 15 months, the patient had less than a 2-degree curvature and has been out of the brace for 3 months. Conclusion: The in-brace overcorrection probably resulted in the exceptional outcome. Successful techniques of scoliosis management are proving to be those that incorporate reversing in a 3-dimensional way a scoliosis patient's posture and spine. (This is a conference presentation abstract and not a full work that has been published.)

\section{Postconcussion symptom reduction and return to play following chiropractic intervention: A case report}

Harold Olson, Michael Tunning, Ron Boesch

Objective: The purpose of this study is to describe the use of chiropractic intervention in the management and return to play of a student athlete with postconcussion syndrome. Clinical Features: A 14-year-old-male hockey player presented to a chiropractic clinic with postconcussion symptoms. He was diagnosed with a concussion 13 days prior. The patient presented with a constant occipital headache with a pain rating of $8 / 10$, upset stomach, blurry vision with concentration, difficulty remembering names, nausea, dizziness, balance problems, "foggy feeling," difficulty with concentration, difficulty with memory, fatigue, confusion, drowsiness, and irritability. Prior to this consultation, he had been following return-to-play guidelines, and progress was being reported via IMPACT testing scores. However, his scores were not improving and remained poor. Intervention and Outcomes: Chiropractic manipulative therapy, myofascial release, instrument-assisted soft tissue technique, and therapeutic exercises were provided in 5 treatments spanning 20 days. The patient followed up each treatment with IMPACT testing. At the conclusion of the treatments, the patient's IMPACT testing scores had improved, and the patient was returned to play. Conclusion: This 14year-old male responded to chiropractic intervention 
following a concussion he sustained while playing hockey. (This is a conference presentation abstract and not a full work that has been published.)

\section{Chiropractic extremity adjusting resolves de Quervain's disease: A case series \\ Paul Otto, Tracy Olson}

Introduction: De Quervain's disease is common among people who repetitively use their wrists at work, in sporting events, playing video games, and/or with smartphone use. The tendons of both the abductor pollicis longus and the extensor pollicis brevis becoming fibrous and degenerating, along with the presence of adhesion in the dorsal compartment, are stated to be the underlying cause of de Quervain's disease. There are many additional treatment options for the treatment of de Quervain's disease, including injections, surgery, and physical therapy. Chiropractic management of 2 patients with severe wrist pain is described. Clinical Features: Case 1 is a 23-year-old male patient with severe wrist pain from repetitive-motion microtraumas, and case 2 is a 42-year-old female secretary with severe bilateral wrist pain. Finklestein's orthopedic testing produced a positive finding in both cases. Intervention and Outcome: Both of the patients were provided radial-ulnar articulation extraspinal adjustment and spinal adjusting. Resolution of wrist pain occurred following 4 months and 2 months, respectively. Conclusion: Chiropractic management, which included both spinal- and extremity-adjusting protocols, has resolved 2 cases diagnosed with de Quervain's disease. (This is a conference presentation abstract and not a full work that has been published.)

\section{Do chiropractic students purchase and read textbooks? A survey of current chiropractic students}

Mark T. Pfefer, Jon D. Wilson, John R. Dobson III, Christopher P. Voll, William Rutherford, Angela R. Segovia, Stephan R. Cooper

Background: Current chiropractic students are enrolled in a heavy load of course work and are asked to purchase and read a number of new textbooks each trimester. Little has been published on reading habits and book purchasing among chiropractic students. Objective: To survey doctor of chiropractic program (DCP) students on reading habits and book purchasing behaviors at 1 institution. Methods: An institutional review board-approved survey was distributed to a convenience sample of chiropractic students at 1 institution. The survey questions were developed by a group of faculty and students to explore reading habits and book purchasing among chiropractic students. Results: A total of 292 surveys out of 377 were completed for a response rate of $77 \%$. Close to $79 \%$ of students stated that they study almost exclusively by using PowerPoint slides provided by the instructors, and only $3 \%$ of respondents stated that they study almost exclusively by textbooks. A majority (77\%) of students purchased 2 or fewer textbooks during the current trimester. Conclusion: Based on these descrip- tive data, DCP students are not likely to read assigned readings from textbooks. Students also purchase textbooks at a very low rate. (This is a conference presentation abstract and not a full work that has been published.)

\section{Ankylosing spondylitis mimicking spasmodic torticollis: A case report}

Lynn Pownall, Stephen Grande, Elizabeth Rokitka

Background: Recent research has shown that early diagnosis of ankylosing spondylitis (AS) leads to increased treatment effectiveness. Objective: The purpose of this study is to introduce a case report of a patient who presented to a chiropractic office with an atypical presentation of AS with spasmodic torticollis (cervical dystonia). Methods: A 26-year-old male presented to a private chiropractic practice with complaints of neck stiffness with decreased cervical range of motion. Based on a clinical exam, negative imaging, and negative HLA B-27, the initial diagnosis was spasmodic torticollis. Results: Short-term improvements to manipulation, deep tissue massage, proprioceptive neuromuscular facilitation, and electromyographic-guided Botox injections were documented. Magnetic resonance imaging 12 and 24 months later revealed sacroiliitis with erosions of the pelvis and thickening of the mid- and upper cervical anterior longitudinal ligament, confirming the diagnosis of AS. A trial of Remicade was initiated. Discussion: AS remains difficult to diagnose in early stages of the disease. Current diagnostic criteria for AS continue to place an emphasis on the lower back with little emphasis on the cervical spine. Conclusion: Further research on expanding the criteria for diagnosis of atypical cases of AS may lead to earlier diagnosis and treatment. (This is a conference presentation abstract and not a full work that has been published.)

\section{An association between low back pain and cigarette smoking}

Dewan Raja, Bahar Sultana

Introduction: We analyzed the pertinent literature regarding low back pain and cigarette smoking. We reviewed 23 articles published in peer-reviewed journals. We looked for the strengths and weaknesses of the methodologies and put together a qualitative assessment derived from the interpretable searched articles. Objective: To present a systematic literature review to explore the evidence displaying the increased prevalence and incidence of low back pain among cigarette smokers. Summary: Millions of patients visit chiropractors and other health care providers to get relief from back pain. Many of the health care providers and their patients do not understand the link between low back pain and cigarette smoking. Results: Of the 23 studies we reviewed, 8 articles described intervertebral disc degeneration as a cause of low back pain. Eleven of the articles included bone cell degeneration as the cause of low back pain. The remaining studies are nonspecific about the cause of low back pain. Most of the reviewed literature featured cross-sectional studies. Three of the 
23 studies were prospective. We attempted to identify the confounding factors related to back pain. Conclusion: This analysis reveals a strong association between cigarette smoking and low back pain. (This is a conference presentation abstract and not a full work that has been published.)

\section{Clinician-specific faculty development program Robert Rectenwald}

Purpose: This is program under development to be included in the existing quarterly faculty and staff development program. It will be tailored to the needs of the clinician and deliver the most current information in clinical practice methods and pedagogy to enable them to more effectively facilitate the learning process of the student interns. Methods: A survey of clinicians was distributed to determine faculty needs. The committee for faculty development approved a proposal for a clinician-specific component to be added to the facultywide development program. Topics were proposed for a pilot. The initial program was presented, and the 2 nd is in planning. Outcomes: A faculty survey showed a favorable opinion of the presentation. Conclusion: Although interns complete didactic course work covering most aspects of chiropractic management, the learning that occurs during the experience of patient care in the clinic system is the most significant component of the clinical educational process. The faculty clinician facilitates this important learning process. As the profession evolves and expands, faculty clinicians require continuing professional development. For clinician faculty development to be most effective, a specific development program is necessary. (This is a conference presentation abstract and not a full work that has been published.)

\section{Early poststroke functional neurological treatment restores function in a patient with hemiparesis: A case report}

Paula Rhodes, Kelsey Brenner

Objective: This case highlights the application of functional neurological treatment in a poststroke case and the results experienced with a patient who had recently experienced stroke. Clinical Features: A 57year-old female presented for evaluation and treatment of left-sided hemiparesis sustained subsequent to a right-sided middle cerebral artery infarct approximately 30 days prior. She exhibited spastic circumduction gait, utilizing a cane for ambulation, spastic paresis of her left hand, loss of sensation on the left side of her face and body, deficit of short-term memory, fatigue, and depressed mood. Intervention and Outcome: Functional neurological treatment was employed twice a week, including left-sided sensory stimulation of upper and lower extremities, supine cross-crawling gait training, mirror-box therapy, and specific eye exercises. Motor control of the left fingers and hand was restored. Motor control and sensation of the left side of her tongue was regained. Near-complete resolution of all signs and symptoms of hemiparesis was noted, with improvement noted in fatigue and mood. Conclusion: Early interven- tion with functional neurological therapies brought rapid resolution of hemiparesis. Rapid resolution of motor and sensory deficits in this case highlights the importance and effectiveness of early functional neurological intervention in cases of stroke. (This is a conference presentation abstract and not a full work that has been published.)

\section{Chiropractic in the age of Ebola: A systematic review of infectious disease prevention and recommendations for chiropractors}

Robert Rowell, Josefina Torres, Karen Goodell

Background: The 2014 Ebola virus disease (EVD) outbreak is one of the worst in history. Cases have occurred in the United States. Given the nonspecific nature of symptoms, other infectious diseases must be considered. Without foreign travel, likely differentials are influenza and pneumonia. Objective: Since chiropractors will see influenza and pneumonia, we conducted a systematic review for literature on preventing infectious disease spread in chiropractic offices. Methods: We searched the Index to Chiropractic Literature, PubMed, Cinahl, AMED, AltHealth Watch, Academic Search Premier, and Health Source Nursing/Academic Edition databases and the Centers for Disease Control (CDC) website. Results: Our search yielded 10 articles and 1 ACC-RAC abstract on infectious organisms, sanitizing chiropractic tables, and hand washing but nothing on preventing the spread of disease between patients. Discussion: We recommend influenza vaccine, respiratory hygiene and cough etiquette, masking and separating symptomatic patients, following previously published table sanitizing and hand hygiene recommendations, and advising ill workers to stay home. Conclusion: No current guidelines exist for preventing the spread of infection between patients with diseases such as influenza or the extremely unlikely EVD. We recommend that the chiropractic profession follow the CDC recommendations. (This is a conference presentation abstract and not a full work that has been published.)

\section{The use of fine arts to improve visual diagnostic skills in chiropractic education}

Drew Rubin

Introduction: Yale University Medical School started bringing their 1st-year medical students to an art museum. The thought was to increase their visual literacy and create better diagnosticians. Most Ivy League medical schools have since followed suit. The author implemented a similar format in a chiropractic educational setting, incorporating visual study of fine art and bringing art into the classroom setting via the Internet. Methods: Two elective classes were given the same pre- and posttests, which included a 10 -question Likert-type scale questionnaire and a pre- and posttest of 2 pictures of children with childhood diseases. The test group was shown fine art paintings and participated in a 5-minute-per-painting discussion over a 5-week period. The control group received the pre- and posttest only, but no paintings were shown. Results: Students in 
the test group had improved posttest grades in their visual diagnostic abilities compared to the control group. Conclusion: Fine arts are an excellent addition to the diagnosis classroom, and this study provided evidence that the use of fine arts in a chiropractic education increased visual literacy and diagnostic skill. (This is a conference presentation abstract and not a full work that has been published.)

\section{Kinetic gait evaluation of 3 patients with foot and ankle injuries before and after a limited protocol of chiropractic care}

Brent Russell, Ronald Hosek, Kathryn Hoiriis

Introduction: There has been little formal gait study of chiropractic care. This report describes 3 patients with foot and ankle injuries, seen as part of a university research lab's ongoing investigation into gait analysis as an outcome measure. Methods: Patients completed a Lower Extremity Functional Scale questionnaire; walking gait was evaluated with an instrumented treadmill before and after the 1st treatment visit and a subsequent visit. Passive movement restrictions were photographed. Patients received handheld instrument or high-velocity, low-amplitude adjustments of the foot and ankle, secondarily for the spine or pelvis, along with stretching or transverse massage. Variability of gait cycle parameters and forces was analyzed using coefficients of variation (CVs). The university's institutional review board approved the study. Results: There were symptomatic and functional improvements and increased motion. Precare, some measures had small CVs, indicating low precare variability. Postcare, decreases of many CVs suggested more consistent walking; however, some CVs increased, and others showed little change. Conclusions: For these participants, some aspects of gait became less variable following chiropractic care; however, the results were mixed. This study contributes to the development of the use of gait analysis as an outcome measure for chiropractic care. Additional investigation is needed. (This is a conference presentation abstract and not a full work that has been published.)

\section{Management of chronic postconscussive central and periphereral symptomotology using chiropractic functional neurologic treatments: A case report}

Brian Sass, Susan Esposito

Objective: This case study illustrates the treatment outcome of a patient with persistent central and peripheral symptoms following a mild traumatic brain injury using chiropractic adjustments in conjunction with brain-based therapies. Clinical Features: A 70year-old female presented for evaluation and treatment with chronic complaints of neck pain, low back pain, balance problems, and dysesthesia. The patient reported that both feet were constantly cold and wet. She also experienced heart arrhythmia, gait problems, and transient dysarthria. Romberg testing revealed 5 seconds of stability. The patient had no perception of vibration below the knee on the right side and on the entire left foot. Heart arrhythmia was evidenced every 3-4 beats. Interventions/Outcomes: Chiropractic adjustments, gaze stability exercises, multiplanar movements of the right lower and upper extremity, vibrational therapy, chair rotations, and eye movement exercises were utilized for treatment. After 10 treatment sessions, the patient regained vibratory sensation on both feet. Stability time was increased to 30 seconds. Dysesthesia significantly decreased. Conclusion: The rapid improvement of sensory disturbances and balance indicate that brain-based treatment can be effective in the management of persistent postconcussive symptoms. These results promote the need for further research. (This is a conference presentation abstract and not a full work that has been published.)

\section{Collaborative care for a patient with complex low back pain and long-term tobacco use: A case report}

Michael Seidman, Robert Vining, Stacie Salsbury

Background: Few examples of interprofessional collaboration by chiropractors and other health care professionals are available. This case report describes an older adult with complex low back pain and long-standing tobacco use who received collaborative health care in a clinical trial conducted with ethics board approval. Case Description: A 65-year-old female retired office worker presented with chronic back pain. Imaging findings included disc extrusion and spinal stenosis. Multiple comorbidities and the complex nature of this case substantiated the need for multidisciplinary collaboration. Intervention: A doctor of chiropractic and a doctor of osteopathy provided collaborative care based on patient goal setting and supported by structured interdisciplinary communication, including record sharing and telephone consultations. Chiropractic and medical interventions included spinal manipulation, exercise, tobacco reduction counseling, analgesic use, nicotine replacement, dietary and ergonomic recommendations, and stress reduction strategies. Conclusion: Collaborative care facilitated active involvement of the patient and resulted in decreased radicular symptoms, improvements in activities of daily living, and tobacco use reduction. Coordinated treatment efforts appeared to result in greater care effectiveness and efficiency in this case. (This is a conference presentation abstract and not a full work that has been published.)

\section{A multidisiplinary approach to work-related musculoskeletal discomfort utilizing a behavior- based ergonomic training program for computer users in a chiropractic college: A research pilot study}

Charles W. Sherrod, Dale F. Johnson, Shawn T. Sherrod

Background: One hundred and sixty million office workers in the United States and two thirds of the developed countries use computers on a daily basis. Objectives: Investigate the effectiveness of implementing an ergonomics training program in a population of computer users in a chiropractic college. Methods: A 
quasi-experimental pilot study on a population $(n=26)$ of computer users working at a chiropractic college. Subjects were randomized into 3 cohorts (comparator online training group, on-site ergonomic training group, and on-site ergonomic training group with surveillance). Assessments were performed with Testing for Ergonomics Knowledge, the Rand SF-36, the Cornell Musculoskeletal Discomfort Questionnaire (CMDQ), and the Transtheoretical Model for Behavior Change (TMBC) algorithm at baseline and 6 weeks. Results: Overall, Knowledge, the Rand SF-36, and the CMDQ showed improvements. However, the K-Form data demonstrated the most definitive changes in symptom reduction and the TMBC algorithm for direction of change to adopt safer work habits. Discussion: Utilizing a multidisciplinary approach for the empowerment of this population of computer users with behavior-based training provided regulatory compliance and enhanced safety and usability. Conclusions: The interventions were effective in improving subjects' ability to interact comfortably and efficiently with their computer workstations. However, a robust population is necessary to avoid bias and confounding while attaining significance. (This is a conference presentation abstract and not a full work that has been published.)

\section{The benefits of an integrated approach to the management of temporal mandibular dysfunction: A case study}

Peter Shipka

Objective: The purpose of this study is to discuss the management and outcome of temporal mandibular joint dysfunction (TMD/TMJ) utilizing an integrated approach that includes acupuncture and chiropractic care. Introduction: $\mathrm{TMD} / \mathrm{TMJ}$ is the term used to describe acute or chronic dysfunction of the joint between the temporal bone and the mandible. This dysfunction may involve the muscles of mastication, nerves, tendons, ligaments, bones, connective tissues, and teeth. Clinical Features: A 43-year-old female presented to a chiropractic clinic complaining of severe jaw, headaches, and neck pain attributed to an injury that occurred 14 years previously. The "lockjaw" had been present for more than 6 weeks and had restricted her jaw opening to less than $18 \mathrm{~mm}$. Intervention and Outcome: An initial session of acupuncture and chiropractic manipulation provided significant improvement, and a series of follow-up visits saw a serious reduction of symptoms and open distances. Dental appliance modifications were also done. Conclusion: Chiropractors who see TMD/TMJpatients should consider an integrated and holistic approach to TMD management. (This is a conference presentation abstract and not a full work that has been published.)

The effects of multimodal chiropractic care on pain and disability in a patient diagnosed with Ehlers-Danlos syndrome-hypermobility type: A case report

Richard Strunk, Mark Pfefer, Kamraan Husain
Background: Ehlers-Danlos syndrome-hypermobility type (EDS-HT) is a hereditary connective tissue disorder defined by joint hypermobility, abnormal skin signs, reoccurring joint dislocations, chronic pain, and a positive family history. Objective: The purpose of this study is to describe the clinical response to multimodal chiropractic treatment of a patient diagnosed with EDS-HT and chronic pain. Methods/Clinical Features: A 22-year-old white female presented with severe chronic neck and low back pain, headaches, and bilateral hand pain and stiffness. The patient was diagnosed with EDS-HT and primarily received lowforce joint manipulative therapy and soft tissue treatment intermittently over 21 months concurrently with care from a rheumatologist. Multiple outcome questionnaires were administered, including but not limited to the Headache and Neck Disability Indices and the Oswestry Low Back Questionnaire. Results: The patient demonstrated clinically meaningful improvements in the outcome questionnaires from baseline to 21 months. Discussion: EDS-HT is a challenging condition to manage, but chiropractic care may be helpful. Conclusion: A description of a patient diagnosed with EDS-HT and managed with multimodal chiropractic care is presented. EDS-HT presents unique treatment challenges, leading us to need more research on this topic. (This is a conference presentation abstract and not a full work that has been published.)

\section{The impact of chiropractic Activator adjustments on mental rotation and line judgment reaction time: A pilot study}

Stephanie Sullivan, Rebecca Shisler Marshall, Lawrence

Hansen, John Markham, Tim Guest

Background: Quality of life and maintenance of selfcare abilities are of high importance. Of value are interventions that can maintain navigational abilities and awareness of body location. Chiropractic from its very foundation has been reported to impact the central nervous system; however, research regarding the specific attributes of improvement is needed. Objective: This pilot study was designed to assess the impact of chiropractic adjustments on spatial cognitive abilities while testing the methodology through assessment in a healthy population. Methods: Using a double-blind, randomized study design, 16 participants underwent 3 computerized tasks ( 1 simple reaction time and 2 visuospatial assessments) before and after receipt of a sham or Activator basic adjustment. Results: Slight improvement was noted for the mental rotation and judgment line orientation tasks; however, no statistically significant difference was evident in either task. Discussion: While statistical relevance was not achieved for this study, the data reflected modest improvement in visuospatial task performance. Further, the methods used in this study were refined for use in future trials. Conclusion: Through impact on the brain's visuospatial capabilities, chiropractic demonstrates potential as a health care solution for the aging population. (This is a conference presentation abstract and not a full work that has been published.) 


\section{Are our students engaging in self-reflection?}

\section{Dorrie Talmage}

Background: Self-reflection is an important tool to use to aid in the development of clinical reasoning skills and the skills necessary to be lifelong learners. Yet are our students engaging in reflective practices? Objective: The purpose of the study was to determine how much time students spent engaging in self-reflection and whether that time changed after exposure to several reflective activities. Methods: A self-reflection survey was administered to 9 consecutive 8th-term classes both at the start and again at the end of the trimester. During the trimester, the students were exposed to activities that encouraged reflection. A paired t test was performed to analyze the survey results. Institutional review board approval was received. Results: The means for all items on the postsurvey was higher than the presurvey. Statistical significance $(p<.05)$ was found on 3 of the 5 items and on all items combined. Discussion: Postsurvey results indicate the students spent more time in self-reflection after the experiencing more patient interactions and course activities. Conclusion: Selfreflection is important to clinical practice, but our educators need to spend time training our students to reflect. (This is a conference presentation abstract and not a full work that has been published.)

\section{Improvement in motor coordination in a senior patient following chiropractic care}

Beatrice Tapia-Borges

Background: Motor coordination is essential to perform our activities of daily living. It involves rhythm, timing, and balance. Balance issues are now used to determine the start and progress of a multitude of conditions. Abnormal motor coordination contributes to falls, which in the elderly population are common and an important health concern. Objective: To see if manual cervical adjustments, using a functional neurology protocol, will improve motor coordination. Methods: A 61-year-old asymptomatic female volunteered for this study. A pretest consisted of a brief examination and computerized testing. The patient then received 10 cervical adjustments. After the 10th adjustment, a posttest was given. Results: Both examination findings, 3 of 6 posturography scores for stability, and all 14 metronome testing scores improved. Discussion: The patient's scores improved significantly. The patient's motor coordination, including rhythm, timing, and balance, with movement improved in all areas. It would be worthwhile to see if these results are reproducible in a larger study. Conclusion: The results of this case indicate that further study with these strategies, for cases of poor balance with senior patients, would be valuable. (This is a conference presentation abstract and not a full work that has been published.)

The deserted library: Students' perceptions of the
library as supporting their academic
achievement-Interpretive case study
Chabha Hocine Tepe

Background: Utilization of library services has been a topic of considerable attention in recent years. It has become contingent on librarians to evaluate library performance and demonstrate the value of library resources and services through documentation and assessment. Objective: To evaluate students' perception of library resources and services through qualitative survey research. Methods: This was an institutional review board-approved investigation. A convenience sample of 15 consenting students participated in semistructured interviews on their perceptions of library services. Individual 10-20-minute interviews were conducted in a private setting during the 1st 2 weeks of November 2013. The author, an experienced librarian, conducted, recorded, and transcribed interviews. Results: Fewer students are satisfied than dissatisfied with the library, particularly with access, resources, and services. A consensus of the interview data shows that the library needs to modernize technology, update physical space, train staff, and enhance virtual platforms to facilitate information access and retrieval. Conclusions: The consensus of students' opinions was that library services need improvement, specifically better access to the physical space, easier navigation of the virtual website (the virtual space of the website is difficult to navigate), and enhanced technology to access library resources. (This is a conference presentation abstract and not a full work that has been published.)

\section{Information literacy self-efficacy and information literacy knowledge of 1st-, 2nd-, and 3rd-year chiropractic students}

Rodger Tepe

Background: Information literacy is a necessary component for chiropractic evidence-based education (EBE) and evidence-based practice (EBP). Little is known about information literacy (IL) self-efficacy (ILSE) and IL knowledge (ILK) among chiropractic students, educators, or practitioners. Purpose: To measure the ILSE and ILK of 1st-, 2nd-, and 3rd-year chiropractic students. Methods: A convenience sample of 396 1st-, 2nd-, and 3rd-year chiropractic students completed a 25 -question ILSE survey and a 25-question ILK test that were based on the American College Research Libraries (ACRL) IL standards. Both instruments have been shown to have acceptable psychometric properties. Results: Significant differences and trends were found between years 1, 2, and 3 ILK means for all 5 ACRL IL standards with year $3>$ year 2 and year 2 $>$ year 1 . No differences were found for ILSE between years 1, 2, and 3. Significant differences were found between mean ILK scores and ILSE tertiles. Conclusion: The finding that 3rd-year students had higher ILK scores than 2nd- and 3rd-year students argues for early IL education in chiropractic curricula. Assessment of IL for chiropractic students, educators, and practitioners is needed to support chiropractic EBE and EBP. (This is a conference presentation abstract and not a full work that has been published.) 


\section{Quantitative assessment of changes in brain activity after a chiropractic adjustment}

Daniel Tuttle, Jerry Hochman, Stephanie Sullivan, Ronald Hosek

Background: The effects of chiropractic adjustments on brain function is a largely understudied area, perhaps due to the lack of cost-effective, objective measures of brain function. Quantitative electroencephalography (qEEG) is a technique that allows for an in-depth analysis of brain activity and may provide a costeffective method for studying chiropractic's impact. Objective: This institutional review board-approved work was undertaken to determine the feasibility of using qEEG to assess brain electrical activity before, during, and after a chiropractic adjustment. Methods: A female patient aged 33 years received a preliminary qEEG assessment. One week later, a follow-up qEEG was conducted before and after receiving a chiropractic adjustment. Results: Analysis using LORETA z scores showed a $2.27-\mathrm{SD}$ increase in values in the delta $(1 \mathrm{~Hz})$ band. Raw value changes with value of $p<.05$ were increased at more sites postadjustment as compared to preliminary assessment. Discussion: Changes in qEEG were markedly more significant following a chiropractic adjustment as compared to changes seen over a 1-week period with no intervention. Conclusion: While the results demonstrate definite qEEG changes following adjustment, further work must be done to determine the most effective way to utilize this technology. (This is a conference presentation abstract and not a full work that has been published.)

\section{Transient quadriplegia in a high school athlete Michael VanNatta}

Objective: To describe chiropractic management of a young athlete with transient quadriplegia, emphasizing back-to-physical-activity considerations. Clinical Features: A 17-year-old male with neck pain and bilateral numbness, tingling, and upper arm and hand weakness (known as a "stinger" injury, which is nerve pinch injury of the upper arm that can also involve the brachial plexus) sought chiropractic care. He reported having recent trauma to his cervical spine region following a football practice blocking drill. Intervention and Outcome: The primary treatment modality used was chiropractic cervical chair supine manipulation designed to improve intersegmental joint dysfunction. The patient had 8 treatments over a 5 -week period. His bilateral arm and hand numbness gradually resolved, and his neck pain improved from $8 / 10$ to $1 / 10$ as measured by Borg scale. Conclusion: The patient responded favorably to chiropractic care, which resulted in decreased neck pain, and the patient was excused from further physical contact sports activity. (This is a conference presentation abstract and not a full work that has been published.)

\section{Challenges in patient recruitment and retention in clinical trials: Is there a need for interprofessional collaboration?}

Sivarama Prasad Vinjamury
Background: Patient recruitment and retention are crucial for successful completion of clinical studies. Objective: To describe the challenges in patient recruitment in clinical trials in general and share the methods that were adopted at our institution to overcome these challenges. Methods: Retrospective evaluation of recruitment data documented during clinical trials. This was supplemented with a literature review of research articles related to recruitment and retention in clinical trials. Results: Literature in this area indicates that the recruitment and retention rates are affected by both patient and investigator/research factors. Duration and nature of the study, inclusion/exclusion criteria, patient expectation/incentive, and patient's unwillingness to take certain treatments (eg, herbal preparation, manipulation, and so on) are some of the factors that influence patient recruitment. In our clinical trials, collaboration with MDs, disease-specific support groups, associations, and referrals from local hospitals were the most effective recruitment methods with very minimal dropouts, as were simplifying the informed consent process, periodic research team meetings, and excluding patients who are unlikely to comply with protocol-addressed recruitment and retention issues effectively. Conclusion: Careful planning and allocation of appropriate resources for recruitment at the beginning of a clinical trial will address the recruitment issues in a clinical trial. (This is a conference presentation abstract and not a full work that has been published.)

\section{Development and implementation of a wiki site at a chiropractic college}

Paul Wanlass, Anupama Kizhakkeveettil, Michael Sackett

Objective: To describe the process of development and implementation of a clinical education wiki site and the students' satisfaction and experiences using this site. Methods: A committee was formed to develop criteria to identify the best computer hardware, software, and camera equipment. The criterion was to ensure compatibility with our existing technology. An institutional review board-approved, anonymous survey was conducted for student cohorts in each year of the chiropractic program to evaluate students' satisfaction and experiences using this site. Results: After considering the criterion, the wiki software was installed on the school server. A phased plan was developed and is currently being implemented. The wiki pages were created to supplement the students' laboratory and classroom education in the areas of physical examination, neurological and orthopedic testing, adjustive technique, soft tissue therapy, palpation, and rehabilitative care. The results of the student survey reveal that the majority of students find the wiki site helpful and easy to use. Conclusions: The development and implementation of a clinical education wiki site is an encouraging approach for improving the educational programs of a chiropractic college. (This is a conference presentation abstract and not a full work that has been published.) 
Impact of justice on organizational and supervisor trust at chiropractic colleges

Dan Weinert, Dustin Derby

Background: Trust has been linked to increased job performance, organizational commitment, goal commitment, organizational citizenship behavior, and decreased intention to quit. Perceptions of justice are an important determinant of trust within an organization. Objectives: Our purpose was to explore the relationship between justice and trust within the chiropractic college setting. We examined what, if any, are the relationships between the forms of justice (distributive, procedural, and interpersonal) and supervisor and organizational trust. Methods: Participants were full-time college faculty employed at US chiropractic colleges and Canadian Memorial Chiropractic College responding to an electronic survey. Instrument validation included exploratory and confirmatory factor analysis. Data analysis included structural regression modeling and structural equation modeling path analysis. Results: Organizational justice was extremely important, accounting for $80 \%$ of the variance in organizational trust. Within this study population, perceptions of interpersonal, procedural, and distributive justice all had significant, positive relationships with faculty members' trust in their supervisor and in their organization. Differences in impact were noted among the forms of justice. Discussion and Conclusions: The findings are important, as organizational and supervisor trust impact many other important variables within the workplace. (This is a conference presentation abstract and not a full work that has been published.)

\section{Diagnostic ultrasound findings in adhesive capsulitis: A report of 2 cases}

Aaron Welk, Norman Kettner

Background: Adhesive capsulitis (AC) is a frequently encountered pain syndrome most often affecting perimenopausal females. The imaging findings of AC are well documented with magetic resonance (MR) imaging, but a comprehensive review of ultrasound (US) features of AC has not been published. Objective: To describe the ultrasound imaging features of adhesive capsulitis. Methods: Two patients were referred for US with a clinical diagnosis of AC. Ultrasound imaging was performed per a standardized protocol with supplemental views of the inferior glenohumeral ligament. A comprehensive analysis of the ultrasound imaging findings was performed. Results: Findings at US included thickening of the glenohumeral joint capsule demonstrated in both patients along with evidence of shoulder complex inflammation. The US imaging findings were consistent with previously published MR imaging evidence of AC. Discussion: $\mathrm{AC}$ is a chronic pain syndrome typically involving the shoulder of perimenopausal females. US is a viable option to confirm the clinical suspicion of $\mathrm{AC}$ and exclude other diagnoses that may result in shoulder pain with limited range of motion. Conclusion: US imaging of $\mathrm{AC}$ was confirmed in 2 cases. The US imaging of $\mathrm{AC}$ may be a viable alternative for conventional MR imaging. (This is a conference presentation abstract and not a full work that has been published.)

\section{Chiropractic student attitudes regarding radiology use: Survey development and validation}

Jon D. Wilson, Mark T. Pfefer, D. Jason Qualls, Angela B. Segovia

Background: Most chiropractic colleges throughout the world use clinical practice guidelines to make decisions on radiographic usage. Although differences in usage between private clinics and college clinics have been documented, limited investigations have been done to measure chiropractic student attitudes regarding radiology use. Methods: An approach was taken to construct and evaluate the validity and reliability of a newly developed survey instrument. This included review by experts, followed by pretesting with a sample chiropractic student population. The final version of the survey was evaluated for reliability using a test-retest and alternate form protocol. The results were analyzed for consistency using an intraclass correlation coefficient. Results: Item-level and scale-level content validity scores obtained from 9 experts were greater than the established threshold levels for acceptable. Face validity and survey critique were provided by 66 students who completed the survey. Reliability among 59 respondents demonstrated moderate to high agreement on a majority of items. Conclusion: The survey instrument appears to have good face validity. All individual items and the survey instrument as a whole appear to have an acceptable level of content validity. All items except 1 appear to have acceptable levels of reliability. (This is a conference presentation abstract and not a full work that has been published.)

\section{Long-term chiropractic management of progressive adolescent idiopathic scoliosis: A case study}

Alan Joshua Woggon

Idiopathic scoliosis is the most common spinal deformity in adolescents, 1 affecting approximately $2 \%-4 \%$ of children $10-16$ years of age. Generalized full-spine adjustments, heel lifts, and postural counseling do not appear to be effective in reducing the severity of mild scoliosis. This study presents the results of a long-term chiropractic treatment plan, developed specifically for scoliosis patients, in a female with adolescent idiopathic scoliosis. The patient was initially diagnosed with idiopathic scoliosis in 2003 at 11 years of age; the Cobb angle was measured at 25 degrees. The patient received generalized chiropractic care, which was not effective in preventing progression. In 2009, the patient traveled out of state to receive care at a private chiropractic clinic that had developed a scoliosis-specific chiropractic treatment protocol; radiographs were taken, and the Cobb angles were measured to be 79 degrees in the thoracic spine and 67 degrees in the lumbar spine. From the initial 
presentation in 2009 to the most recent $\mathrm{x}$-ray taken in 2014, the thoracic Cobb angle changed from 79 to 42 degrees, and the lumbar Cobb angle changed from 67 to 43 degrees, representing a $46.8 \%$ reduction in the thoracic Cobb angle and a $35.8 \%$ reduction in the lumbar Cobb angle. (This is a conference presentation abstract and not a full work that has been published.)

Magnetic resonance imaging-measured change in intracranial flow following upper cervical chiropractic care of a migraine headache subject: A case report

H. Charles Woodfield III, D. Gordon Hasick

In an attempt to study changes in cerebral circulation, it was discovered that patients with migraine headaches exhibit an increase in intracranial compliance index (ICCI), following an intervention defined by the National Upper Cervical Chiropractic Association (NUCCA). Using phase contrast magnetic resonance imaging (PC-MRI), the primary objective of this case report was measuring changes in the intracranial compliance index (ICCI) from baseline to week 4, then again at week 8 , following a NUCCA atlas intervention. This subject is 1 of 11 from an observational pilot study, exhibiting an unexpected decrease in ICCI instead of increases. This subject was recruited through a neurology-based specialist referral clinic. She signed consent forms, completed baseline migraine-specific outcomes, and then was examined by a neurologist. Presence of atlas misalignment indicated inclusion, allowing baseline PC-MRI assessment. Subjects received NUCCA care for 8 weeks. Follow-up imaging occurred at weeks 4 and 8 . Neurologist exit interviews allowed for final outcomes collection. The primary outcome, ICCI unexpectedly decreased from 5.04 to 3.87. This subject reported great improvement in migraine headache symptoms while demonstrating significant improvement in migraine-specific patientreported outcomes. A decrease in ICCI, while expected to increase, creates questions to answer through future study. (This is a conference presentation abstract and not a full work that has been published.)

\section{Interexaminer reliability in analysis of orthogonal radiographs}

\section{H. Charles Woodfield III, John F. Hart, Peter B. Jacquemin}

Background: An assessment for making important clinical decisions requires due diligence in investigation in reliability of its use. Little evidence appears in the indexed literature positively reporting examiner reliability of the orthogonal radiographic analysis. Objective: The objective was determining interexaminer reliability between 2 National Upper Cervical Chiropractic Association board-certified practitioners analyzing orthogonal films. Methods: Before-correction film sets, screened for acceptability, were randomly sent to 2 examiners with analysis data mailed to a data manager. Double-entered verified data were forwarded for statistical analysis. Statistical analysis allowed data interpretation through percent agreement, intraclass correlation coefficient (ICC), and Bland-Altman limitsof-agreement plots. Results: Percent agreement of atlas laterality was $96.1 \%(244 / 254)$ with $94.5 \%(240 / 254)$ agreement on side of rotation. ICCs for laterality were $0.907,95 \%$ confidence interval $(\mathrm{CI})(0.882,0.926)$ (consistency model) and $0.906,95 \%$ CI $(0.881,0.926)$ (agreement model) $(n=254)$. For rotation, ICCs were $0.849,95 \%$ CI $(0.811,0.880)$ (consistency model) and $0.850,95 \%$ CI $(0.812,0.881)$ (agreement model) $(n=$ 254). Bland-Altman plots revealed 5.9\% (14/254) of paired observations outside $95 \%$ agreement limits for laterality and, for rotation, $6.2 \%$ (15/254) of measurements. Discussion: Frequent consensus training possibly improves reliability. The convenience sample of radiographs used could create possible bias in overestimating ICCs. Conclusion: Based on statistical analysis, excellent (near perfect) reliability was found between 2 NUCCA board-certified practitioners analyzing orthogonal films. (This is a conference presentation abstract and not a full work that has been published.) 